Brichacek, A. L., Neill, J. T., Murray, K. (2018). The effect of basic psychological needs and exposure to idealised Facebook images on university students' body satisfaction. Cyberpsychology: Journal of Psychosocial Research on Cyberspace, 12(3), article 2. http://dx.doi.org/10.5817/CP2018-3-2

\title{
The effect of basic psychological needs and exposure to idealised Facebook images on university students' body satisfaction
}

\author{
Anna L. Brichacek, James T. Neill, \& Kristen Murray
}

Centre for Applied Psychology, School of Psychology and Counselling, Faculty of Health, University of Canberra, Canberra, Australia

\begin{abstract}
Exposure to ideal body types in the media has been consistently linked to reduced body satisfaction. Images posted on social networking sites may also impact body satisfaction by portraying idealised standards of physical attractiveness promoted by peers. This study draws on self-determination theory to examine whether satisfaction of basic psychological needs (autonomy, competence, and relatedness) protects against the negative effect of viewing Facebook images depicting an ideal body type on body satisfaction. Female $(n=141)$ and male $(n=48)$ university students were randomly assigned to view either a body-ideal image or a travel image presented on a mock Facebook profile. Viewing body-ideal imagery resulted in significantly lower body satisfaction compared to viewing travel imagery $(d=-0.37)$. Satisfaction of the needs for autonomy and competence predicted higher baseline body satisfaction; however, none of the psychological needs protected against the negative effect of viewing body-ideal imagery on body satisfaction. Limitations included brief exposure to a single Facebook image and use of a convenience sample. Future research may benefit from measuring body image-specific rather than general psychological need satisfaction to predict state changes in body satisfaction.
\end{abstract}

Keywords: Body satisfaction; Facebook; social networking sites; psychological needs; self-determination theory

\section{Introduction}

Body image is an important part of self-concept, encompassing perceptual, evaluative-affective, cognitive, and behavioural aspects of body shape, weight, size, and function (Tiggemann, 2004). Body satisfaction, the evaluativeaffective component of body image, is associated with physical and psychological health, with low body satisfaction predictive of poorer health outcomes such as low self-esteem and higher rates of depression and disordered eating (Paxton, Neumark-Sztainer, Hannan, \& Eisenberg, 2006). According to the tripartite influence model (Thompson, Heinberg, Altabe, \& Tantleff-Dunn, 1999), parents, peers, and the mass media provide three sociocultural pathways through which body image ideals are communicated and reinforced, and comparisons with and internalisation of ideals can lead to decreased body satisfaction. Recently, researchers have considered the influence of newer forms of online and social media, which provide additional avenues for disseminating body image ideals (Brown \& Tiggemann, 2016; Cohen, Newton-John, \& Slater, 2017; Holland \& Tiggemann, 2016).

\section{Social Networking Sites and Body Satisfaction}

Social media differs to traditional media in scope, accessibility, and pervasiveness (Kaplan \& Haenlein, 2010). Social networking sites (SNSs) may be particularly influential for body satisfaction as they are largely image-based and 
allow selective construction of an idealised self (Strano, 2008). Furthermore, peers provide an important source of social comparison for young people possibly explaining why appearance comparisons on SNSs are associated with lower body satisfaction than in-person comparisons, and more negative mood than traditional media comparisons, in female university students (Fardouly, Pinkus, \& Vartanian, 2017).

Facebook is the most popular SNS among young adults (Smith \& Anderson, 2018), and users report lower body satisfaction than non-users (Stronge et al., 2015). Whilst brief (10-minute) exposure to Facebook in general had no impact on body satisfaction (Fardouly, Diedrichs, Vartanian, \& Halliwell, 2015), female students who viewed a Facebook News Feed depicting attractive women were less satisfied with their own appearance than students who viewed less-attractive women (Kim \& Park, 2016). In a systematic review of 20 studies Holland and Tiggemann (2016) found that engaging in photo-based activities on SNSs was particularly detrimental for body satisfaction, and there is preliminary evidence to support a causal link between viewing body-ideal images on Instagram and reduced body satisfaction among young women and men (Tamplin, McLean, \& Paxton, 2018; Tiggemann \& Zaccardo, 2015). Few other studies have examined the effect of viewing idealised Facebook images on body satisfaction and the existing research is limited to females. Further experimental research is therefore needed to clarify causal mechanisms in gender diverse samples.

Additionally, there has been little theoretically grounded research into psychological factors that protect against SNS effects on body satisfaction. Individual difference characteristics may act as internal resources that mitigate potential harms of viewing idealised Facebook images (Kim \& Park, 2016). For example, being critical of commercial content on SNSs and having high self-esteem, low appearance schemas, and lower tendencies to engage in social comparison buffers against the effect of SNSs on body satisfaction in females, suggesting that not all individuals are susceptible to negative consequences (Ahadzadeh, Sharif, \& Ong, 2017; Kim \& Park, 2016; Tamplin et al., 2018). One factor considered important in explaining differences in perceived sociocultural pressures around body image is self-determination (Matusitz \& Martin, 2013; Pelletier, Dion, \& Lévesque, 2004).

\section{Self-Determination Theory}

According to self-determination theory (SDT), individuals have an innate tendency to regulate behaviour based on choice and interest and, under need-supportive conditions, will grow and flourish (Deci \& Ryan, 2000). SDT proposes that this movement towards self-determination and optimal psychological well-being relies on the satisfaction of basic psychological needs for autonomy, competence, and relatedness (Vansteenkiste \& Ryan, 2013). Autonomy refers to experiencing volition and self-determination in behaviour; competence involves perceiving that one has the necessary skills to interact effectively within the environment; and relatedness is feeling accepted by, and connected to, others (Chen et al., 2015). When psychological needs are satisfied, behaviour is regulated by intrinsic values rather than being contingent on external validation (Vansteenkiste \& Ryan, 2013). As such, satisfaction of basic psychological needs may serve a protective function against sociocultural messages about what constitutes an ideal body image (Pelletier et al., 2004; Tylka \& Kroon Van Diest, 2015).

There are two pathways through which psychological need satisfaction may be protective. First, psychological need fulfilment may promote greater overall body satisfaction, as self-evaluations are more likely to be based on personal preference rather than societal expectations (Vansteenkiste \& Ryan, 2013). Thøgersen-Ntoumani, Ntoumanis, and Nikitaras (2010) found that higher psychological need satisfaction predicted less body dissatisfaction and drive for thinness in 350 female adolescents. Similarly, in a large sample of physically active males $(N=552)$, individuals who reported that their psychological needs were being actively undermined by pressuring, controlling, or unsupportive environments experienced greater muscle dissatisfaction (Edwards, Tod, Molnar, \& Markland, 2016). Another study found that only autonomy satisfaction positively predicted body satisfaction in male and female fitness instructors when controlling for age and BMI (Thøgersen-Ntoumani \& Ntoumanis, 2007). These studies suggest that psychological need fulfilment is related to increased body satisfaction; however, it is unclear whether certain needs (e.g., autonomy) may be of greater importance.

Psychological needs may also offer a protective function by reducing the impact of external threats to body image, such as viewing idealised Facebook images. Individuals whose psychological needs are satisfied are more likely to define their self-worth in terms of stable internal factors rather than physical appearance (Pelletier et al., 2004). Instead of interpreting idealised Facebook images as a source of pressure, they may selectively disregard 
information that is incongruent with their values and shift attention towards more important personal qualities. Female university students with high general self-determination were protected against the negative effect of viewing four minutes of thin-ideal video advertisements on body satisfaction (Mask \& Blanchard, 2011). Similarly, social support buffered against body dissatisfaction following exposure to fashion magazines in adolescent females (Stice, Spangler, \& Agras, 2001). Feeling self-determined and supported are conditions that arise out of satisfying the needs for autonomy, competence, and relatedness (Vansteenkiste \& Ryan, 2013), and thus basic psychological need fulfillment may be an underlying factor that protects against the harmful effects of viewing body image ideals.

\section{The Present Study}

This study aims to investigate whether the potentially protective role of psychological need satisfaction transfers to university students when viewing Facebook images depicting an ideal body type. Feeling self-determined, effective, and connected may be especially important when viewing idealised images of a gender and ethnicitymatched peer given the increased relevance and perceived attainability of the ideal (Fardouly et al., 2017). Consistent with previous research (Tiggemann \& Zaccardo, 2015), it is hypothesised that viewing a body-ideal Facebook image will result in lower body satisfaction compared to viewing a travel image (H1). According to SDT, satisfaction of basic psychological needs promotes more authentic and favourable self-evaluations, and thus it is hypothesised that autonomy, competence, and relatedness will each predict higher baseline body satisfaction (H2). Psychological needs (autonomy, competence, and relatedness) may also protect against external body image pressures (Pelletier et al., 2004), and are therefore expected to positively predict change in body satisfaction following exposure to body-ideal imagery $(\mathrm{H} 3)$.

\section{Method}

\section{Participants}

Participants were 189 university students (75\% female), aged between 18 and 58 years $(M=22.6, M d n=21.0, S D=$ 6.6), with self-reported Body Mass Index (BMI) ranging from 16.2 to $44.4 \mathrm{~kg} / \mathrm{m}^{2}(M=23.9, M d n=22.8, S D=5.1)$. Participants were recruited from first-year psychology (88\%) and other Faculty of Health units (12\%) at the University of Canberra. Participants identified as Caucasian (71\%), Asian (13\%), Australian Aboriginal or Torres Strait Islander (5\%), African (3\%) or other ethnicities (7\%). The majority of participants (93\%) were active Facebook users.

\section{Design}

The study employed a between-subjects experimental design, with a two-level independent variable (image type: body-ideal, travel) and a continuous dependent variable (body satisfaction). Additional predictors were psychological need satisfaction (autonomy, competence, and relatedness), with participant age, gender, BMI, and ethnicity used as covariates where they correlated with the dependent variable.

\section{Materials}

The following measures were presented as part of a larger survey investigating SNS usage, sociocultural pressures around body image, health, and subjective well-being. The survey was implemented online using Qualtrics (2016). Only materials relevant to the current study are described here.

Demographics and social networking site usage. Participants indicated their age, gender, ethnicity, current weight and height (used to calculate BMI), and their typical frequency (Never, Daily, Weekly, Fortnightly, or Monthly or less) and duration (hours and minutes) of SNS usage.

Images. Photographs that met strict inclusion criteria, including that the picture was of a single person, represented an ideal body type for that gender and ethnicity (e.g., thin ideal for females, muscular/lean ideal for males; Ahern, Bennett, Kelly, \& Hetherington, 2010; Cafri, Strauss, \& Thompson, 2002), and at least half of the body 
was visible, were selected from online open-source databases (e.g., Wikimedia Commons). From an initial pool of 18 eligible photographs, the first and second authors selected images that depicted the ideal body type for each gender and ethnic group (male and female images for Caucasian, Asian, and Other ethnicities) resulting in a total of six body-ideal images. Where gender and ethnicity differed, it was ensured that other aspects of the images remained constant (e.g., percentage of body visibility, camera angle, and image quality). A travel image depicting a cityscape with unidentifiable people in the foreground was selected as the control (see Appendix).

Photographs were embedded within a Facebook profile screenshot and displayed with the written instruction, "Imagine one of your friends just posted this picture of themselves online". To ensure that participants attended to the image, they were asked an open-ended question, "Please describe your thoughts and feelings in response to seeing this photo on Facebook". Two participants, whose responses indicated they had not attended to the task, were excluded from analyses.

Need satisfaction. The 12 satisfaction items from the Basic Psychological Need Satisfaction and Frustration Scale (Chen et al., 2015) were used to measure the extent to which autonomy, competence, and relatedness needs were met. Items such as, "I feel a sense of choice and freedom in the things I undertake." (autonomy), "I feel capable at what I do." (competence), and "I feel connected with the people who care for me and for whom I care." (relatedness) were responded to on a 5-point scale ( 1 = not true at all, $5=$ completely true). Item scores were averaged to calculate composite scores for autonomy (4 items), competence (4 items), and relatedness ( 4 items), with higher scores indicating greater need fulfilment. All subscales demonstrated good internal consistency (see Table 1).

Body satisfaction. The 6-item Body Image States Scale (Cash, Fleming, Alindogan, Steadman, \& Whitehead, 2002) was used to measure participants' current ("at this moment") body satisfaction. Responses on a 9-point scale were averaged to create baseline and post-exposure body satisfaction scores, with higher values indicating greater satisfaction. Four filler items assessing health, employment, relationships, and current living arrangements were included to disguise the true purpose of the study. The scale demonstrated excellent internal consistency at both time points (see Table 1).

\section{Procedure}

The study was approved by the University Human Research Ethics Committee (Protocol number 16-32). First-year undergraduate students were invited to participate in the study on "Social media, health, body perceptions, and subjective well-being" in return for academic credit or entry into a prize-draw for one of two AU\$50 shopping vouchers. The online survey took approximately 20 minutes to complete.

After providing demographic information and completing baseline measures, including body satisfaction and basic psychological need satisfaction, participants were randomly assigned to view a body-ideal or travel image using a 4:1 experimental to control ratio. Unequal randomisation was used to maximise power in subsequent analyses. Participants in the body-ideal condition ( $n=152,70 \%$ female) viewed a single image that was matched to their gender and ethnicity. Participants in the control condition ( $n=36,76 \%$ female) viewed the travel image. Following image exposure, participants completed the post-exposure body satisfaction measure. Upon completion, participants were provided with debrief information and awarded course credit.

\section{Results}

There was less than 5\% missing data for all variables, and Little's MCAR test indicated that data was missing completely at random. Statistical assumptions were met for all analyses.

A one-way analysis of covariance examined the effect of viewing a body-ideal versus travel image on body satisfaction ( $\mathrm{H} 1)$, controlling for BMI, gender, and baseline body satisfaction. Groups did not differ on BMI $(F(1,186)$ $=0.09, p=.769)$ or baseline body satisfaction $(F(1,186)=0.21, p=.647)$. However, there was a significant betweengroup difference in post-exposure body satisfaction $(F(1,182)=3.93, p=.049, d=-0.37)$. Participants who viewed the body-ideal image had significantly lower body satisfaction $(M=4.68, S D=1.80)$ compared to those who viewed the travel image $(M=5.07, S D=1.45)$, a small effect according to Cohen (1992). 
A hierarchical multiple linear regression (MLR) was used to examine the extent to which autonomy, competence, and relatedness explained baseline body satisfaction $(\mathrm{H} 2)$. BMI and gender, entered in Step 1, accounted for a significant $19 \%$ of variance in body satisfaction $(F(2,184)=22.12, p<.001)$. Autonomy, competence, and relatedness, entered in Step 2, explained a further $25 \%$ variance $(\Delta F(3,181)=26.32, p<.001)$. Autonomy and competence were small to moderate positive predictors of baseline body satisfaction in the final model (adjusted $R^{2}=0.42, F(5,181)=28.29, p<.001$; see Table 2$)$.

Finally, a hierarchical MLR was used to test the extent to which autonomy, competence, and relatedness predicted change in body satisfaction as a result of viewing a body-ideal image (H3). Baseline body satisfaction and covariates (BMI and gender) were entered in Step 1 and predicted a significant $83 \%$ of variance in post-exposure body satisfaction $(F(3,147)=244.11, p<.001)$. Autonomy, competence, and relatedness, entered in Step 2, did not significantly predict any additional variance $\left(\Delta R^{2}=0.00, \Delta F(3,144)=0.38, p=.765\right)$, indicating that psychological needs were not protective against exposure to body-ideal imagery.

Table 1. Means, Standard Deviations, Internal Consistencies, and Correlations Between Study Variables.

\begin{tabular}{|c|c|c|c|c|c|c|c|c|c|c|}
\hline \multirow{2}{*}{$\begin{array}{l}\text { Variable } \\
\text { Post body } \\
\text { satisfaction }\end{array}$} & \multicolumn{2}{|c|}{$\begin{array}{l}\text { Baseline body } \\
\text { satisfaction }\end{array}$} & \multicolumn{2}{|c|}{$\begin{array}{l}\text { Post body } \\
\text { satisfaction }\end{array}$} & \multicolumn{2}{|c|}{ Autonomy } & \multicolumn{2}{|c|}{ Competence } & \multicolumn{2}{|c|}{ Relatedness } \\
\hline & .91 & $\star \star \star$ & - & & & & & & & \\
\hline Autonomy & .47 & $\star \star \star$ & .43 & $\star \star \star$ & - & & & & & \\
\hline Competence & .44 & $\star \star \star$ & .39 & $\star \star \star$ & .57 & $\star \star \star$ & - & & & \\
\hline Relatedness & .25 & $\star \star$ & .22 & $\star \star$ & .47 & $\star \star \star$ & .47 & $\star \star \star$ & - & \\
\hline$M(S D)$ & 4.89 & $(1.61)$ & 4.75 & (1.74) & 3.65 & $(0.65)$ & 3.63 & $(0.73)$ & 3.97 & $(0.81)$ \\
\hline Cronbach's a & .87 & & .92 & & .74 & & .84 & & .88 & \\
\hline
\end{tabular}

Note: ${ }^{*} p<.05 ; * \star p<.01 ; * \star * p<.001$.

Table 2. Regression Coefficients and Squared Semi-partial Correlations for Basic Psychological Needs as Predictors of Baseline Body Satisfaction ( $\mathrm{n}=187$ ).

\begin{tabular}{|c|c|c|c|c|}
\hline Step & Variable & $B\left[\mathrm{Cl}_{95^{a}}\right]$ & $\beta$ & $s r^{2 \mathrm{~b}}$ \\
\hline
\end{tabular}

Step 1

$\begin{array}{lllll}\text { Gender } & -0.66[7.79-10.44] & -0.18 & * * & 0.03 \\ \text { BMI } & -0.13[-0.17--0.09] & -0.41 & * \star * & 0.16\end{array}$

Step 2

\begin{tabular}{|c|c|c|c|c|}
\hline Gender & $-0.84[-1.26--0.43]$ & -0.23 & $\star \star \star$ & 0.05 \\
\hline BMI & $-0.11[-0.15--0.08]$ & -0.36 & $\star \star \star$ & 0.13 \\
\hline Autonomy & $0.68[0.33-1.03]$ & 0.28 & $\star \star \star$ & 0.05 \\
\hline Competence & $0.58[0.28-0.89]$ & 0.27 & $\star \star \star$ & 0.04 \\
\hline Relatedness & $0.07[-0.19-0.34]$ & 0.04 & & 0.00 \\
\hline
\end{tabular}

Note: ${ }^{\mathrm{a}} \mathrm{Cl}_{95}=95 \%$ confidence interval; ${ }^{\mathrm{b}} s r^{2}=$ Squared semi-partial correlation.

$\star p<.05 ; * \star p<.01 ; * \star * p<.001$. 


\section{Discussion}

This study analysed the effect of brief exposure to body-ideal Facebook imagery on body satisfaction and tested the protective role of basic psychological needs. As predicted, viewing a Facebook image depicting an ideal body type resulted in lower body satisfaction compared to viewing a travel image. Satisfaction of autonomy and competence psychological needs explained $25 \%$ of the variance in baseline body satisfaction. Contrary to predictions, however, satisfaction of psychological needs did not protect against declines in body satisfaction following exposure to body-ideal imagery.

The present study strengthens previous experimental research demonstrating that exposure to gender-matched images of attractive individuals on SNSs can have short-term adverse effects on university students' body satisfaction (Kim \& Park, 2016; Tamplin et al., 2018; Tiggemann \& Zaccardo, 2015). It should be noted that effects were small and, while statistically significant, were close to the critical cut-off. Stronger effects may occur if participants were to view a greater number of images over a longer timeframe, as typically experienced when using Facebook. Nevertheless, the effect was similar in size to that of short-term exposure to idealised images through television and magazines (Grabe, Ward, \& Hyde, 2008) indicating that SNSs are at least as detrimental to body satisfaction as conventional media (Cohen \& Blaszczynski, 2015; Fardouly et al., 2017).

Results further highlight the importance of basic psychological need fulfilment for body satisfaction. Autonomy and competence, the two self-focused needs, positively predicted body satisfaction, while relatedness did not emerge as a significant predictor. This finding is consistent with the positive relationship between autonomy and body satisfaction observed in fitness instructors (Thøgersen-Ntoumani \& Ntoumanis, 2007), but differs somewhat from Thøgersen-Ntoumani et al.'s (2010) finding that autonomy, competence, and relatedness were equivalent predictors of body satisfaction in adolescent females. Relatedness may be more important for body satisfaction during adolescence than adulthood because adolescents' body image is more strongly influenced by family and peers (Stice et al., 2001). Whilst psychological needs and body satisfaction appear to be related, the cross-sectional nature of this data limits our ability to make causal inferences.

The finding that satisfaction of basic psychological needs did not protect against the negative effect of viewing body-ideal Facebook imagery on body satisfaction contrasts previous studies on traditional media (Mask \& Blanchard, 2011; Stice et al., 2001). Mask and Blanchard (2011) measured general self-determination and thus it may be this particular motivational outcome of psychological need satisfaction that is protective against sociocultural pressures. Alternatively, psychological needs may be protective against body-ideals presented in traditional media but not Facebook. Ideals promoted through traditional media for commercial purposes may be easily perceived as incongruent with internal values and disregarded, whereas peers' ideals may be harder to differentiate from personal preferences and even highly autonomous individuals may experience momentary reductions in body satisfaction. For example, when sociocultural pressures included messages from family, friends, and dating partners, self-determination did not protect against internalisation of the thin-ideal in female university students (Pelletier et al., 2004). Nevertheless, conceptual differences between the current study and previous experimental studies, most notably of which was controlling for baseline body satisfaction in the current study and sample characteristics, limit our ability to make direct comparisons. The results presented here suggest that protective effects may occur because self-determined individuals approach sociocultural pressures with initially higher levels of body satisfaction.

An important limitation of the current study was that participants were exposed to a single image in a mock Facebook context. More powerful effects may occur with increased exposure or if participants viewed and interacted with images of peers from their own social networks. Whilst steps were taken to disguise the true purpose of the study, participants were not asked whether they were aware of the study aims, and thus demand characteristics cannot be ruled out. Generalisability is also limited by use of a convenience sample of predominantly female Caucasians attending one Australian university. Replication is needed to establish whether the current findings extend to males and diverse ethnic groups. Nevertheless, young women are among the highest consumers of SNSs and represent a population for which body image issues are particularly prevalent (Mond et al., 2013). 
Despite its limitations, this study contributes to understanding body satisfaction in university students and has implications for future research. Findings support the need to increase students' awareness around the impact of Facebook on mental health and address SNS-specific content, such as motivations behind image posting, in interventions designed to reduce the negative effects of media exposure (McLean, Wertheim, Masters, \& Paxton, 2017). The influential role of SNSs on body satisfaction also points to the potential for platforms such as Facebook to be used to improve body image if body-positive content were promoted, and there is initial research to support the utility of SNSs for this purpose (Slater, Varsani, \& Diedrichs, 2017). Prevention and intervention programs may also benefit from addressing broader aspects of the self, beyond weight and shape, as body satisfaction was related to fulfillment of underlying basic human needs.

Further research could improve ecological validity by increasing the length of exposure and having participants view images of friends whom they contact both online and offline. Furthermore, whilst this study investigated the effects of passive Facebook use, experimental studies examining active involvement (e.g., uploading photos) could provide a more nuanced understanding of how SNSs impact body satisfaction. Research examining temporal relationships between psychological need satisfaction and body satisfaction is needed to determine whether psychological needs could help in the prevention and treatment of body- and eating-related issues. Enquiry into mechanisms through which need satisfaction relates to body satisfaction, for example by promoting intrinsic versus extrinsic values and increasing self-determination, could further advance understanding of SDT in the context of body image. Finally, further research is recommended to clarify whether the protective role of psychological need satisfaction may depend on the exposure context, image source (e.g., uploaded by peers versus celebrities), or participant characteristics.

It is also worth noting that, although this study tested the protective role of global psychological need fulfillment, psychological needs can be conceptualised at the contextual or situational level (Vallerand, 1997). Future research may benefit from exploring psychological need satisfaction as experienced in the domain of body image, as a context-specific measure may be more sensitive to changes in state body satisfaction.

In conclusion, this study found that brief exposure to Facebook images depicting an ideal body type resulted in lower body satisfaction among university students. Satisfaction of psychological needs for autonomy and competence were associated with greater body satisfaction; however, psychological needs were not protective against the short-term negative effects of viewing Facebook imagery on body satisfaction. These findings extend previous literature on the relationship between SNSs and body image and highlight the need for additional research into psychological factors that may protect against adverse outcomes.

\section{Acknowledgements}

Role of Funding Sources: This research did not receive any specific grant from funding agencies in the public, commercial, or not-for-profit sectors.

\section{References}

Ahadzadeh, A. S., Sharif, S. P., \& Ong, F. S. (2017). Self-schema and self-discrepancy mediate the influence of Instagram usage on body satisfaction among youth. Computers in Human Behavior, 68, 8-16.

https://doi.org/10.1016/j.chb.2016.11.011

Ahern, A. L., Bennett, K. M., Kelly, M., \& Hetherington, M. M. (2010). A qualitative exploration of young women's attitudes towards the thin ideal. Journal of Health Psychology, 16, 70-79.

https://doi.org/10.1177/1359105310367690

Brown, Z., \& Tiggemann, M. (2016). Attractive celebrity and peer images on Instagram: Effect on women's mood and body image. Body Image, 19, 37-43. https://doi.org/10.1016/j.bodyim.2016.08.007

Cafri, G., Strauss, J., \& Thompson, J. K. (2002). Male body image: Satisfaction and its relationship to well-being using the somatomorphic matrix. International Journal of Men's Health, 1, 215-231.

https://doi.org/10.3149/jmh.0102.215 
Cash, T. F., Fleming, E. C., Alindogan, J., Steadman, L., \& Whitehead, A. (2002). Beyond body image as a trait: The development and validation of the Body Image States Scale. Eating Disorders, 10, 103-113. https://doi.org/10.1080/10640260290081678

Cohen, J. (1992). A power primer. Psychological Bulletin, 112, 155-159. https://doi.org/10.1037/00332909.112.1.155

Cohen, R., \& Blaszczynski, A. (2015). Comparative effects of Facebook and conventional media on body image dissatisfaction. Journal of Eating Disorders, 3, article 23. https://doi.org/10.1186/s40337-015-0061-3

Cohen, R., Newton-John, T., \& Slater, A. (2017). The relationship between Facebook and Instagram appearancefocused activities and body image concerns in young women. Body Image, 23, 183-187.

https://doi.org/10.1016/j.bodyim.2017.10.002

Chen, B., Vansteenkiste, M., Beyers, W., Boone, L., Deci, E. L., Kaap-Deeder, J., ...Verstuyf, J. (2015). Basic psychological need satisfaction, need frustration, and need strength across four cultures. Motivation \& Emotion, 39, 216-236. https://doi.org/10.1007/s11031-014-9450-1

Deci, E. L., \& Ryan, R. M. (2000). The "what" and "why" of goal pursuits: Human needs and the self-determination of behavior. Psychological Inquiry, 11, 227-268. https://doi.org/10.1207/S15327965PLI1104_01

Edwards, C., Tod, D., Molnar, G., \& Markland, D. (2016). Predicting muscularity-related behavior, emotions, and cognitions in men: The role of psychological need thwarting, drive for muscularity, and mesomorphic internalization. Body Image, 18, 108-112. https://doi.org/10.1016/j.bodyim.2016.06.005

Fardouly, J. Diedrichs, P. C., Vartanian, L. R., \& Halliwell, E. (2015). Social comparisons on social media: The impact of Facebook on young women's body image concerns and mood. Body Image, 13, 38-45. https://doi.org/10.1016/bodyim.2014.12.002

Fardouly, J., Pinkus, R. T., \& Vartanian, L. R. (2017). The impact of appearance comparisons made through social media, traditional media, and in person in women's everyday lives. Body Image, 20, 31-39.

https://doi.org/10.1016/j.bodyim.2016.11.002

Grabe, S., Ward, L. M., \& Hyde, J. S. (2008). The role of the media in body image concerns among women: A metaanalysis of experimental and correlational studies. Psychological Bulletin, 134, 460-476.

https://doi.org/10.1037/0033-2909.134.3.460

Holland, G., \& Tiggemann, M. (2016). A systematic review of the impact of the use of social networking sites on body image and disordered eating outcomes. Body Image, 17, 100-110.

https://doi.org/10.1016/j.bodyim.2016.02.008

Kaplan, A. M., \& Haenlein, M. (2010). Users of the world, unite! The challenges and opportunities of Social Media. Business Horizons, 53, 59-68. https://doi.org/10.1016/j.bushor.2009.09.003

Kim, M., \& Park, W. (2016). Who is at risk on Facebook? The effects of Facebook News Feed photographs on female college students' appearance satisfaction. The Social Science Journal, 53, 427-434.

https://doi.org/10.1016/j.soscij.2016.08.007

Mask, L., \& Blanchard, C. M. (2011). The protective role of general self-determination against 'thin ideal' media exposure on women's body image and eating-related concerns. Journal of Health Psychology, 16, 489-499. https://doi.org/10.1177/1359105310385367

Matusitz, J., \& Martin, J. (2013). The application of self-determination theory to eating disorders. Journal of Creativity in Mental Health, 8, 499-517. https://doi.org/10.1080/15401383.2013.850392 
McLean, S. A., Wertheim, E. H., Masters, J. \& Paxton, S. J. (2017). A pilot evaluation of a social media literacy intervention to reduce risk factors for eating disorders. International Journal of Eating Disorders, 50, 847-851. https://doi.org/10.1002/eat.22708

Mond, J., Mitchison, D., Latner, J., Hay, P., Owen, C., \& Rodgers, B. (2013). Quality of life impairment associated with body dissatisfaction in a general population sample of women. BMC Public Health, 13, 920-931.

https://doi.org/10.1186/1471-2458-13-920

Paxton, S. J., Neumark-Sztainer, D., Hannan, P. J., \& Eisenberg, M. E. (2006). Body dissatisfaction prospectively predicts depressive mood and low self-esteem in adolescent girls and boys. Journal of Clinical Child and Adolescent Psychology, 35, 539-549. https://doi.org/10.1207/s15374424jccp3504_5

Pelletier, L. G., Dion, S. C., \& Lévesque, C. S. (2004). Can self-determination help protect women against sociocultural influences about body image and reduce their risk of experiencing bulimic symptoms? Journal of Social and Clinical Psychology, 23, 61-88. https://doi.org/10.1521/jscp.23.1.61.26990

Qualtrics (2016). Qualtrics [Computer software]. https://qualtrics.com

Slater, A. Varsani, N., Diedrichs, P. C. (2017). \#fitspo or \#loveyourself? The impact of fitspiration and selfcompassion Instagram images on women's body image, self-compassion, and mood. Body Image, 22, 87-96. https://doi.org/10.1016/j.bodyim.2017.06.004

Smith, A., \& Anderson, M. (2018). Social Media use in 2018. Pew Research center. Retrieved from http://www.pewinternet.org/2018/03/01/social-media-use-in-2018/

Stice, E., Spangler, D., \& Agras, W. S. (2001). Exposure to media-portrayed thin-ideal images adversely affects vulnerable girls: A longitudinal experiment. Journal of Social and Clinical Psychology, 20, 270-288. https://doi.org/10.1521/jscp.20.3.270.22309

Strano, M. (2008). User descriptions and interpretations of self-presentation through Facebook profile images. Cyberpsychology: Journal of Psychosocial Research on Cyberspace, 2(2), article 5. Retrieved from https://cyberpsychology.eu/article/view/4212/3253

Stronge, S., Greaves, L. M., Milojev, P., West-Newman, T., Barlow, F. K., \& Sibley, C. G. (2015). Facebook is linked to body dissatisfaction: Comparing users and non-users. Sex Roles, 73, 200-213. https://doi.org/10.1007/s11199015-0517-6

Tamplin, N. C., McLean, S. A., \& Paxton, S. J. (2018). Social media literacy protects against the negative impact of exposure to appearance ideal social media images in young adult women but not men. Body Image, 26, 29-37. https://doi.org/10.1016/j.bodyim.2018.05.003

Thøgersen-Ntoumani, C., \& Ntoumanis, N. (2007). A self-determination theory approach to the study of body image concerns, self-presentation and self-perceptions in a sample of aerobic instructors. Journal of Health Psychology, 12, 301-315. https://doi.org/10.1177/1359105307074267

Thøgersen-Ntoumani, C., Ntoumanis, N., \& Nikitaras, N. (2010). Unhealthy weight control behaviours in adolescent girls: A process model based on self-determination theory. Psychology \& Health, 25, 535-550. https://doi.org/10.1080/08870440902783628

Thompson, J. K., Heinberg, L. J., Altabe, M., \& Tantleff-Dunn, S. (1999). Exacting beauty: Theory, assessment, and treatment of body image disturbance. Washington, DC: American Psychological Association.

Tiggemann, M. (2004). Body image across the adult life span: Stability and change. Body Image, 1, 29-41. https://doi.org/10.1016/S1740-1445(03)00002-0 
Tiggemann, M., \& Zaccardo, M. (2015). "Exercise to be fit, not skinny": The effect of fitspiration imagery on women's body image. Body Image, 15, 61-67. https://doi.org/10.1016/j.bodyim.2015.06.003

Tylka, T. L., \& Kroon Van Diest, A. M. (2015). Protective factors. In L. Smolak \& M. P. Levine (Eds.), The Wiley handbook of eating disorders (pp. 430-444). West Sussex, England: John Wiley and Sons.

Vallerand, R. J. (1997). Toward a hierarchical model of intrinsic and extrinsic motivation. Advances in Experimental Social Psychology, 29, 271-360. https://doi.org/10.1016/S0065-2601(08)60019-2

Vansteenkiste, M., \& Ryan, R. M. (2013). On psychological growth and vulnerability: Basic psychological need satisfaction and need frustration as a unifying principle. Journal of Psychotherapy Integration, 23, 263-280. https://doi.org/10.1037/a0032359 


\section{Appendix}

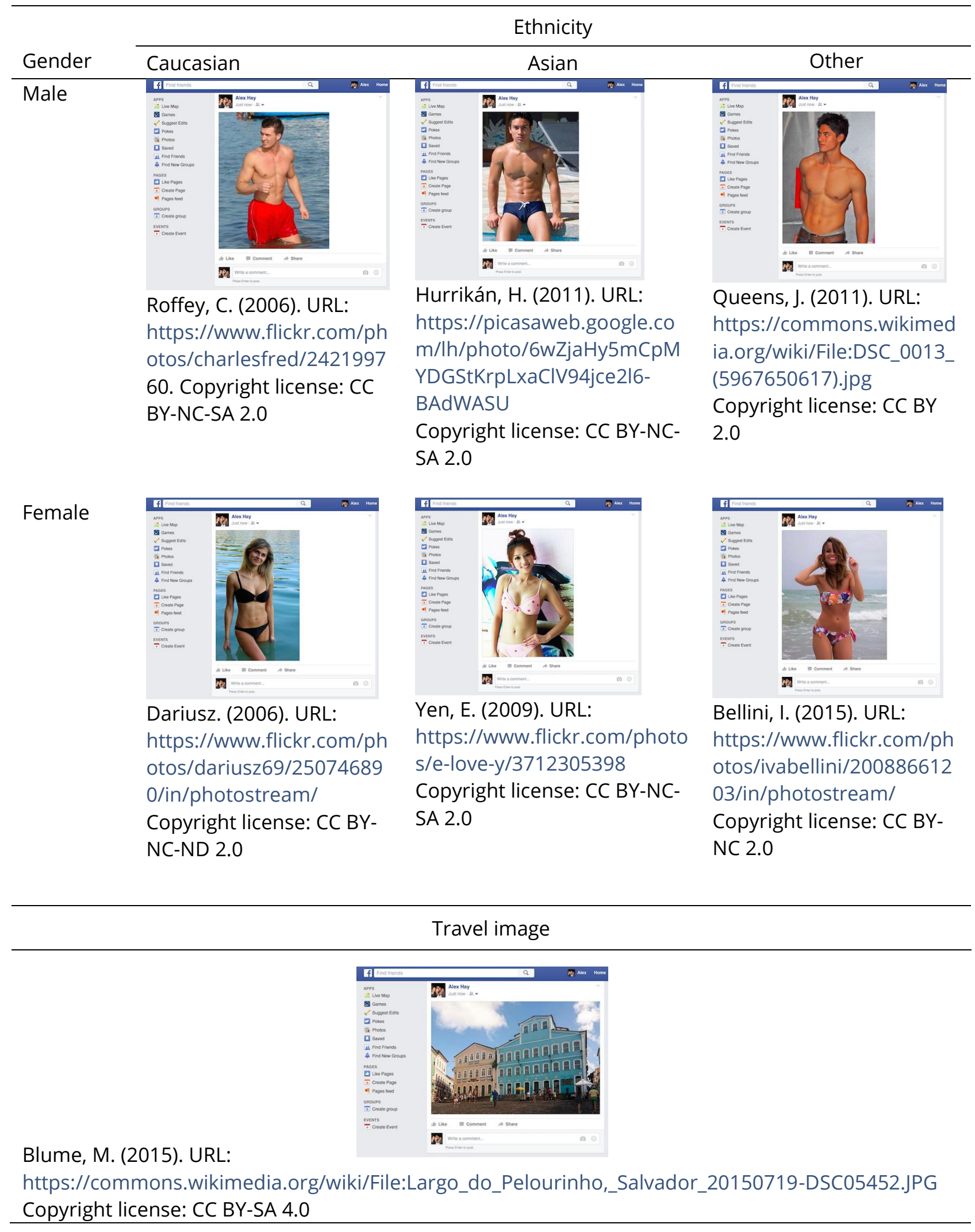

Figure A1. Body-ideal images by gender and ethnicity with source and copyright information. 


\section{Correspondence to:}

Anna Brichacek

Centre for Applied Psychology, School of Psychology and Counselling, Faculty of Health

Locked Bag 1, University of Canbesrra

Australian Capital Territory 2601

Australia

Email: anna.brichacek(at)uni.canberra.edu.au

Editorial record: First submission received on November 30, 2017. Revisions received on August, 21, 2018, and October 24, 2018. Accepted for publication on October 24, 2018.

\section{About Authors}

Anna Brichacek is completing a Doctor of Philosophy in Clinical Psychology at the University of Canberra, Australia. Her research interests include body image, coping and resilience, and acceptance- and mindfulnessbased approaches. Her PhD is specifically examining protective aspects of positive body image that may help to improve eating and health outcomes in young people.

James Neill is an Assistant Professor in the Centre for Applied Psychology at the University of Canberra, Australia. His research interests include positive psychology, social media, adventure-based learning, and measurement of psychological change.

Dr Kristen Murray is a Clinical Psychologist and Assistant Professor in Clinical Psychology at the University of Canberra, Australia. Her research interests in clinical and health psychology include understanding aetiology, assessment, and intervention in body image, obesity, and eating disorders; psychological stress; and interprofessional practice to enhance health and well-being outcomes in the population. 